\title{
ORIGINAL
}

\section{Urinary creatinine excretion is related to short-term and long-term mortality in critically ill patients}

\author{
Lara Hessels * $^{*}$, Niels Koopmans², Antonio W. Gomes Neto³, Meint Volbeda', Jacqueline Koeze', \\ Annemieke Oude Lansink-Hartgring ${ }^{1}$, Stephan J. Bakker ${ }^{3}$, Heleen M. Oudemans-van Straaten ${ }^{4}$ \\ and Maarten W. Nijsten ${ }^{1}$
}

(c) 2018 The Author(s)

\begin{abstract}
Purpose: Patients with reduced muscle mass have a worse outcome, but muscle mass is difficult to quantify in the ICU. Urinary creatinine excretion (UCE) reflects muscle mass, but has not been studied in critically ill patients. We evaluated the relation of baseline UCE with short-term and long-term mortality in patients admitted to our ICU.

Methods: Patients who stayed $\geq 24 \mathrm{~h}$ in the ICU with UCE measured within 3 days of admission were included. We excluded patients who developed acute kidney injury stage 3 during the first week of ICU stay. As muscle mass is considerably higher in men than women, we used sex-stratified UCE quintiles. We assessed the relation of UCE with both in-hospital mortality and long-term mortality.

Results: From 37,283 patients, 6151 patients with 11,198 UCE measurements were included. Mean UCE was 54\% higher in males compared to females. In-hospital mortality was 17\%, while at 5-year follow-up, 1299 (25\%) patients had died. After adjustment for age, sex, estimated glomerular filtration rate, body mass index, reason for admission and disease severity, patients in the lowest UCE quintile had an increased in-hospital mortality compared to the patients in the highest UCE quintile (OR 2.56, 95\% Cl 1.96-3.34). For long-term mortality, the highest risk was also observed for patients in the lowest UCE quintile (HR 2.32,95\% Cl 1.89-2.85), independent of confounders.
\end{abstract}

Conclusions: In ICU patients without severe renal dysfunction, low urinary creatinine excretion is associated with short-term and long-term mortality, independent of age, sex, renal function and disease characteristics, underscoring the role of muscle mass as risk factor for mortality and UCE as relevant biomarker.

Keywords: Creatinine, Urinary creatinine excretion, Muscle mass, Muscle wasting, Sarcopenia, Glomerular filtration rate, In-hospital mortality, Long-term mortality

\section{Introduction}

Muscle mass is an important determinant of the ability of patients in the intensive care unit (ICU) to overcome

\footnotetext{
*Correspondence: lara.hessels@gmail.com

${ }^{1}$ Department of Critical Care, University of Groningen, University Medical Center Groningen, Hanzeplein 1, 9700 RB Groningen, The Netherlands

Full author information is available at the end of the article

Lara Hessels and Niels Koopmans contributed equally.
}

their disease. Sarcopenia (i.e. loss of muscle and function) on ICU admission is an independent risk factor for morbidity and mortality in critically ill patients [1-3]. Although several physical and laboratory indicators of muscle mass have been used in various other patient groups $[4,5]$, muscle mass is difficult to quantify in ICU patients.

Creatinine is the stable end product of creatine. Most creatine is present in muscle and is converted at a steady rate to creatinine. Creatinine is released into the

\section{至 Springer}


circulation and is almost exclusively excreted in the urine [6]. In steady state conditions, urinary excretion will equal creatinine production, irrespective of the serum creatinine concentration. Therefore, measurement of urinary creatinine excretion (UCE) in 24-h urine collections is a widely accepted method for muscle mass estimation in stable outpatient populations [5, 7-9]. In healthy subjects [10] and in patients with wasting conditions or (chronic) renal failure [4, 8], UCE has been associated with long-term mortality.

UCE has not been evaluated in critically ill patients. In our ICU, 24-h urine is routinely and continuously collected to measure UCE. We hypothesized that in critically ill patients baseline UCE, as a reflection of muscle mass, is related with mortality. We analyzed the relation of UCE with short-term and long-term mortality.

\section{Materials and methods}

\section{Study setting, patient selection and outcome}

In this retrospective observational cohort study, we analyzed laboratory measurements of all patients aged 15 years and older who were admitted to our ICU in a university hospital between January 2002 and April 2016. Reason for ICU-admission, age, sex, height, weight and the acute physiology and chronic health evaluation score 4 (APACHE-IV) [11] were recorded. We routinely collect 24-h urine samples as part of standard care at our ICU to determine the measured creatinine clearance. From 00:00 to 24:00 all urine is collected in a large disposable container. Patients who were discharged within $24 \mathrm{~h}$ of ICU admission or for whom no 24-h urine samples were available in the first 3 days after admission (i.e. due to measurement errors in the lab or incomplete 24 -h collections) were excluded. Only 24-h urine samples collected in the first 3 days after ICU admission were analyzed. UCE was determined by multiplying the urinary creatinine concentration in the 24-h urine with the 24-h urinary volume. We did not use weight-adjusted daily UCE, as we do not routinely weigh our patients. The median UCE was calculated for each patient and used for further analyses. Corresponding daily serum creatinine levels were also available. Acute kidney injury (AKI) was assessed for the first 7 days of ICU admission. Patients with acute kidney injury (AKI) stage 3 (i.e. increase of serum creatinine to $>300 \%$ from baseline, or $\geq 354 \mu \mathrm{mol} / \mathrm{L}(4 \mathrm{mg} / \mathrm{dL})$ or requiring renal replacement therapy [12]) during the first 7 ICU days, were excluded because of their inability to produce urine or the unreliability of UCE as RRT interferes with UCE interpretation. Since complete data on urine output were often not available, we only used the serum creatinine based criteria of the KDIGO AKI guideline. We stratified for sex to account for the considerable difference in creatinine excretion resulting from

\section{Take-home message:}

Low urinary creatinine excretion early after ICU admission is a strong independent predictor of both short-term and long-term mortality, underscoring a role of muscle mass as risk factor for mortality. UCE thus constitutes a simple, readily available and relevant prognostic biomarker for critically ill patients.

differences in body weight and composition between men and women [13]. This study was approved by our hospital's medical ethical committee and since it concerned an analysis of anonymized laboratory and clinical data, all collected during standard clinical care, informed consent was not required (METc 2011/132).

\section{Samples}

Urinary and serum creatinine measurements were performed in the hospital's certified central laboratory. Serum creatine kinase activity (CK) measured at ICU day one was also recorded to assess a possible effect of rhabdomyolysis on creatinine. We did not exclude patients with potential rhabdomyolysis. Potential rhabdomyolysis was defined as $\mathrm{CK} \geq 1500 \mathrm{U} / \mathrm{L}$. Cardiothoracic surgery patients were not included in this subgroup. Estimated glomerular filtration (eGFR) was calculated with the Chronic Kidney Disease Epidemiology Collaboration (CKD-EPI) formula [14] with serum creatinine, sex, and age as input variables. Body mass index (BMI) was calculated as weight $(\mathrm{kg}) /$ height $^{2}(\mathrm{~m})$. In order to adjust for acute changes in renal clearance of creatinine we also calculated the estimated creatinine production, as described in the supplementary material file (SMF). Likewise, in the SMF we compared weight-adjusted UCE, i.e. UCE $/ \mathrm{kg}$ with UCE in the predictive models in patients with available baseline body weight measurements.

\section{Outcome}

In-hospital mortality was used as the short-term outcome measure. We performed near-complete long-term follow-up to determine survival status in patients for 5 years after hospital discharge, as recorded in the hospital database and in the municipal mortality registry by January 2018.

\section{Statistical analysis}

Patient characteristics were calculated according to sexstratified UCE quintiles. Data were expressed as mean and standard deviation (SD) when normally distributed or median and interquartile range (IQR) when skewed. A chi-square test for categorical variables and ANOVA for normally distributed continuous variables or a KruskalWallis test for skewed distributed continuous variables was performed to determine variances between patient 
characteristics across UCE quintiles. Missing data were imputed via multiple imputation (see SMF).

To assess associations of UCE with short-term and long-term mortality respectively, multivariable logistic regression and Cox proportional hazards regression analyses were performed. The proportional hazard assumption was verified by inspection of "log-log" plots and by introducing interactions with survival time. UCE was entered as a categorical variable (quintiles) and as a continuous variable (with OR/HR calculated per $5 \mathrm{mmol} / 24 \mathrm{~h}$ UCE decrease). Analyses were first performed in a crude model (model 1: adjusted for sex when UCE was entered as continuous variable). Further analyses cumulatively included adjustment for age (model 2), eGFR (model 3), BMI (model 4) and reason of admission and severity of illness (model 5). For patients discharged alive from the hospital, long-term survival was assessed with Kaplan-Meier survival curves according to the sexstratified UCE quintiles and evaluated with the log-rank test. Patients who were lost to follow-up were censored at that particular time point. Splines were fit by a logistic regression model and a Cox proportional hazards regression model based on restricted cubic splines and adjustments as used in model 5. In secondary analyses, we tested for potential interaction by sex, age, BSA, renal function, disease severity and reason of admission. We also performed separate analyses for patients who developed AKI and for patients who did not develop AKI. Additional subgroup analyses were performed when effect modification was observed or when differences in UCE were expected in patient subgroups. In sensitivity analyses, we investigated for potential bias introduced by imputation, by restricting the dataset to complete cases. As additional sensitivity analysis, we assessed the potential confounding effect of rhabdomyolysis on UCE. Serum CK was log-transformed to adjust for its strongly skewed distribution. The secondary and sensitivity analyses as listed in the results and SMF were adjusted for potential confounders that were included in model 5. $P$ values $<0.05$ were considered significant. Data were analyzed with SPSS 23.0 (IBM Inc. 2016, New York, USA) and $R$. version 3.4.2 ( $R$ foundation for Statistical Computing, Vienna, Austria).

\section{Results}

\section{Patient characteristics and outcome}

Of a total of 37,283 patients, 6151 patients were included. We excluded 28,493 patients because of ICU admission with a duration shorter than $24 \mathrm{~h}$ or incomplete $24-\mathrm{h}$ urine collection. Another 2572 patients were excluded because of AKI stage 3 within 7 days of ICU admission, and finally 67 patients were excluded because of missing serum creatinine levels. In the remaining 6151 patients, a total of 11,198 24-h urine creatinine measurements (i.e. 1.8 measurements per patient) were determined during the first 3 ICU days (Fig. 1). The baseline clinical characteristics of the included patients are summarized in Table 1. Median age of the included patients was $62(50-72)$ years and $62 \%$ were male. Median UCE (IQR) was $54 \%$ higher in men than women, i.e. 12.2 $(9.0-15.7)$ vs. $7.9(6.0-10.1) \mathrm{mmol} / \mathrm{day}(P<0.001)$. The mean UCE was similar on ICU-days 1 and $3(10.8 \pm 5.2$ vs. $11.0 \pm 5.1 \mathrm{mmol} /$ day, $P=0.34)$. Median urinary volume was $1.5 \mathrm{~L}$ (1.01-2.2). Reason for admission differed

\section{7,283 patients admitted to the ICU 2002-2016}

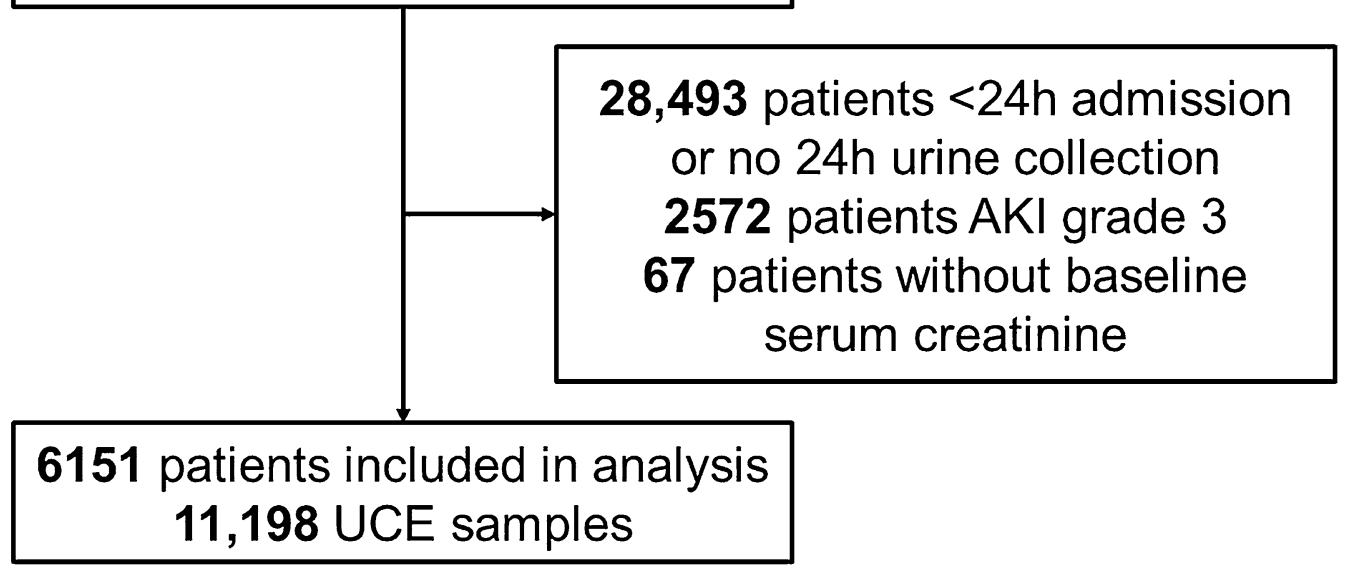

Fig. 1 Flowchart of patients included into the analysis 
Table 1 Patient characteristics and outcome parameters

\begin{tabular}{|c|c|c|c|c|c|c|}
\hline & \multicolumn{5}{|c|}{ UCE sex-stratified quintiles ${ }^{a}$} & \multirow[t]{3}{*}{$P$} \\
\hline & Q1 & Q2 & Q3 & Q4 & Q5 & \\
\hline & $\delta^{\prime} \leq 8.25 ; \uparrow \leq 5.55$ & $\begin{array}{l}\text { o > 8.25-10.9; } \\
\wp>5.55-7.10\end{array}$ & $\begin{array}{l}\text { o > 10.9-13.45; } \\
\wp>7.10-8.55\end{array}$ & $\begin{array}{l}\text { o > 13.45-16.65; } \\
\text { }>8.55-10.50\end{array}$ & $\delta>16.65 ; \uparrow>10.50$ & \\
\hline Included patients & 1228 & 1237 & 1208 & 1240 & 1238 & \\
\hline Male (\%) & $760(62 \%)$ & $770(62 \%)$ & $756(63 \%)$ & $764(62 \%)$ & $764(62 \%)$ & 0.987 \\
\hline Age, years & $67(56-76)$ & $67(58-76)$ & $66(56-73)$ & $60(48-69)$ & $51(38-61)$ & $<0.001$ \\
\hline $\begin{array}{l}\text { Urinary creatinine } \\
\text { excretion, } \mathrm{mmol} / 24 \mathrm{~h}\end{array}$ & $5.3 \pm 2.0$ & $8.4 \pm 1.7$ & $10.6 \pm 2.2$ & $12.9 \pm 2.8$ & $17.2 \pm 4.5$ & $<0.001$ \\
\hline Men & $6.0 \pm 1.9$ & $9.7 \pm 0.7$ & $12.2 \pm 0.7$ & $15.0 \pm 0.9$ & $20.0 \pm 3.1$ & $<0.001$ \\
\hline Female & $4.0 \pm 1.3$ & $6.3 \pm 0.5$ & $7.8 \pm 0.4$ & $9.5 \pm 0.6$ & $12.8 \pm 2.4$ & $<0.001$ \\
\hline $\begin{array}{l}\text { Reason for admission } \\
(\%)\end{array}$ & & & & & & $<0.001$ \\
\hline Medical & $173(14 \%)$ & $129(10 \%)$ & $126(10 \%)$ & $158(13 \%)$ & $153(12 \%)$ & \\
\hline \multicolumn{7}{|l|}{ Surgical } \\
\hline Trauma & $35(3 \%)$ & $48(4 \%)$ & $58(5 \%)$ & $117(9 \%)$ & $279(23 \%)$ & \\
\hline $\begin{array}{l}\text { Abdominal/vas- } \\
\text { cular }\end{array}$ & $288(23 \%)$ & $297(24 \%)$ & $291(24 \%)$ & $315(25 \%)$ & $289(23 \%)$ & \\
\hline Transplantation & $54(4 \%)$ & $66(5 \%)$ & $54(4 \%)$ & $51(4 \%)$ & $18(2 \%)$ & \\
\hline Neurosurgery & $31(3 \%)$ & $32(3 \%)$ & $41(3 \%)$ & $59(5 \%)$ & $86(7 \%)$ & \\
\hline Cardiothoracic & $243(20 \%)$ & $319(26 \%)$ & 347 (29\%) & $286(23 \%)$ & $193(16 \%)$ & \\
\hline Miscellaneous & $408(33 \%)$ & $346(28 \%)$ & 291 (24\%) & $254(21 \%)$ & $220(18 \%)$ & \\
\hline ICU LOS, days & $4.8(2.6-10.1)$ & $4.9(2.7-10.1)$ & $4.1(2.3-8.7)$ & $4.3(2.3-9.6)$ & $4.5(2.5-9.8)$ & 0.004 \\
\hline Hospital LOS, days & $18.1(9.2-34.7)$ & $20.2(12.1-34.2)$ & $17.4(11.2-30.0)$ & $16.9(10.3-28.2)$ & $16.8(10.2-28.7)$ & $<0.001$ \\
\hline APACHE-IV & $73 \pm 27$ & $67 \pm 24$ & $64 \pm 24$ & $58 \pm 24$ & $53 \pm 23$ & $<0.001$ \\
\hline Length, $\mathrm{cm}^{\mathrm{c}}$ & $171 \pm 10$ & $173 \pm 9$ & $174 \pm 9$ & $176 \pm 9$ & $178 \pm 9$ & $<0.001$ \\
\hline Weight, kg ${ }^{\mathrm{d}}$ & $73 \pm 16$ & $75 \pm 14$ & $80 \pm 15$ & $83 \pm 15$ & $90 \pm 18$ & $<0.001$ \\
\hline $\mathrm{BMI}^{\mathrm{C}}$ & $25 \pm 5$ & $25 \pm 4$ & $26 \pm 4$ & $27 \pm 5$ & $28 \pm 6$ & $<0.001$ \\
\hline BSA, $m^{2 c}$ & $1.8 \pm 0.2$ & $1.9 \pm 0.2$ & $2.0 \pm 0.2$ & $2.0 \pm 0.2$ & $2.1 \pm 0.2$ & $<0.001$ \\
\hline Acute kidney injury & $539(44 \%)$ & $414(34 \%)$ & $305(25 \%)$ & $233(19 \%)$ & $218(18 \%)$ & $<0.001$ \\
\hline Stage 1 & $353(65 \%)$ & 301 (73\%) & $229(75 \%)$ & 173 (79\%) & $163(64 \%)$ & \\
\hline Stage 2 & $186(35 \%)$ & $113(27 \%)$ & $76(25 \%)$ & $60(27 \%)$ & 55 (25\%) & \\
\hline $\begin{array}{l}\text { Serum creatinine, } \\
\mu \mathrm{mol} / \mathrm{L}\end{array}$ & $86(56-136)$ & $76(58-115)$ & $73(58-100)$ & 70 (58-93) & $69(58-87)$ & $<0.001$ \\
\hline eGFR (mL/min/1.73m2) & $72(40-102)$ & $82(51-100)$ & $87(61-101)$ & $93(69-107)$ & $100(81-114)$ & $<0.001$ \\
\hline
\end{tabular}

a Urinary creatinine excretion quintiles based on separate quintile intervals for males and females in mmol per day

b Data missing for 1709 (28\%) patients

c Data missing for $1176(19 \%)$ patients

d Data missing for 1173 (19\%) patients

between the quintiles of UCE, with the highest number of trauma patients in the highest UCE quintile.

The median ICU length of stay was 4.6 (2.5-9.7) days, with a total hospital stay of $17.9(10.8-30.7)$ days (Table 1). The median serum creatinine was 73 (57104) $\mu \mathrm{mol} / \mathrm{L}$ and 1709 patients (28\%) developed AKI stage 1 or stage 2 in the first week of ICU stay. Serum creatinine decreased in the first 3 ICU days [day 1: 79 (62-104), day 3: 73 (57-106) $\mu \mathrm{mol} / \mathrm{L}, P<0.001]$. Serum creatinine and incidence of AKI were inversely associated with UCE quintiles. Median eGFR was $92(60-122) \mathrm{mL} /$ min and was positively associated with UCE quintiles. Median follow-up time was 3.7 (2.1-7.6) years with a maximum of 16.1 years. A completeness of follow-up of 85\% was achieved (see Supplementary Methods, SMF).

\section{UCE and short-term mortality}

Overall in-hospital mortality was $17 \%$. In-hospital mortality decreased for the sex-specific quintiles of UCE, from $31 \%$ in the first quintile to $9 \%$ in the fifth quintile 
Table 2 Logistic regression analyses of in-hospital mortality

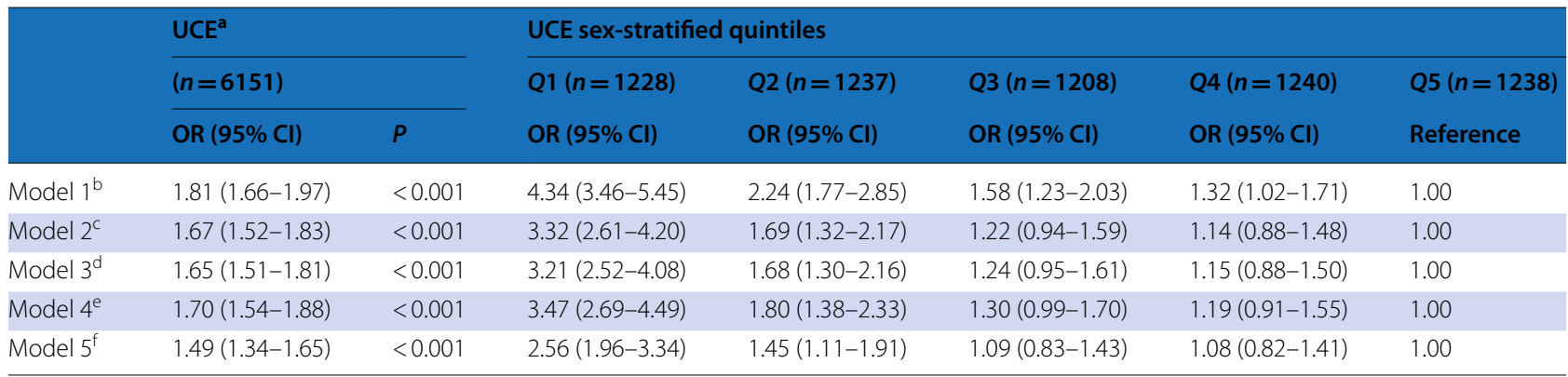

Multivariable logistic regression to assess the association of UCE with in-hospital mortality

a UCE was entered as a continuous variable per $5 \mathrm{mmol} / 24 \mathrm{~h}$ decrease

${ }^{b}$ Model 1: adjusted for sex in continuous analyses, no adjustment for sex-adjusted quintiles

c Model 2: adjusted as for model 1, additionally adjusted for age

d Model 3: adjusted as for model 2, additionally adjusted for kidney function (eGFR CKD-EPI)

e Model 4: adjusted as for model 3, additionally adjusted for body mass index (BMI)

${ }^{f}$ Model 5: adjusted as for model 4, additionally adjusted for severity of illness (APACHE-IV) and reason of admission (trauma vs non-trauma)

$(P<0.001$, Fig. 2a). In multivariable logistic regression analyses with sex-specific quintiles of UCE, there was a 2.4 times increased risk of in-hospital mortality in the lowest sex-specific UCE quintile compared to highest quintile (OR 2.56, 95\% CI 1.96-3.34, $P<0.001$ ), independent of potential confounders (Table 2, model 5). In multivariable logistic regression analyses, with adjustment for sex, UCE expressed as a continuous variable was inversely associated with in-hospital mortality (for each $5 \mathrm{mmol} / 24 \mathrm{~h}$ decrease of UCE: OR $1.81,95 \% \mathrm{CI}$ 1.66-1.97, $P<0.001$; Table 2). This association remained significant (OR 1.49, 95\% CI 1.34-1.65, $P<0.001$ ), independent of potential confounders (Table 2, model 5).
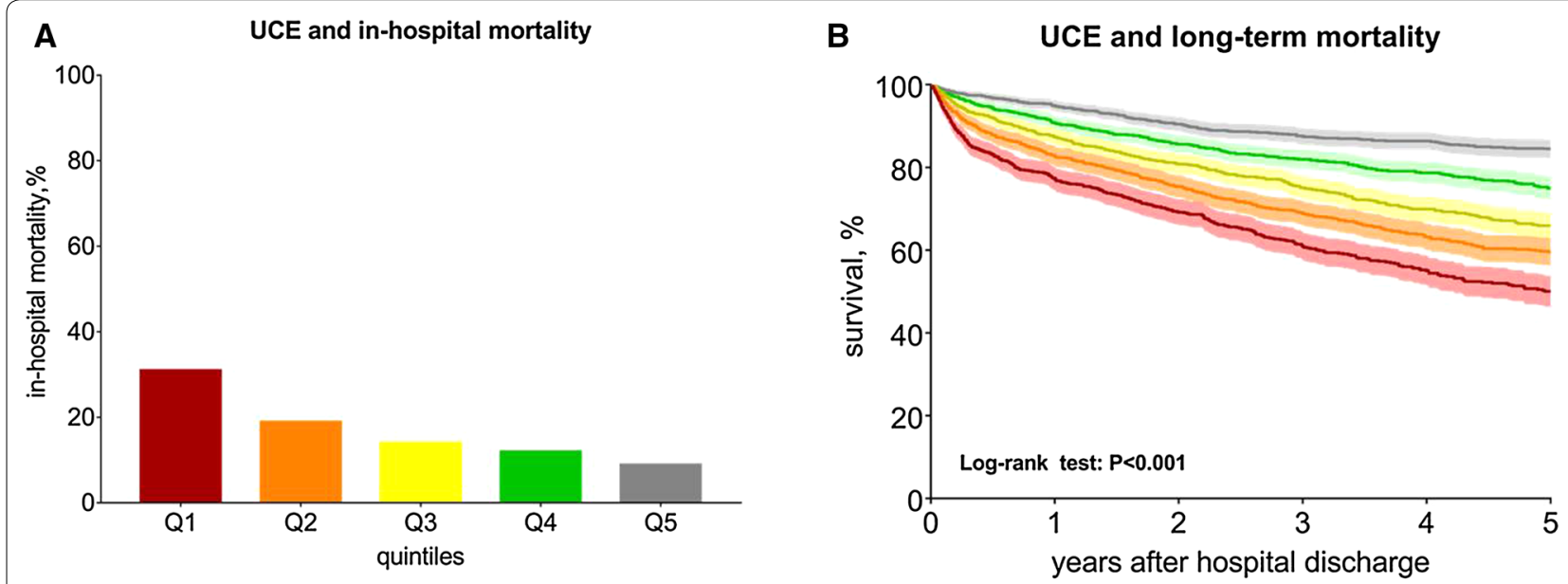

\begin{tabular}{lcccccc}
\multicolumn{2}{l}{ Nrs at risk } & & & & & \\
Q1 & 850 & 658 & 589 & 529 & 495 & 471 \\
Q2 & 1006 & 834 & 759 & 701 & 659 & 636 \\
Q3 & 1040 & 909 & 841 & 790 & 753 & 729 \\
Q4 & 1092 & 991 & 935 & 900 & 875 & 850 \\
Q5 & 1123 & 1066 & 1016 & 986 & 976 & 961
\end{tabular}

Fig. 2 Short-term and long-term mortality as expressed in UCE quintiles. a In-hospital mortality is depicted for the UCE quintiles in percentages. The first quintile represents the lowest UCE, the fifth quintile represents he highest quintile. Corresponding quintile cut-off values are shown in Table 1. In-hospital mortality increased when baseline UCE decreased (chi-square test: $P<0.001$ ). b Kaplan-Meier curves for 5 year survival (with $95 \% \mathrm{Cl}$ ) after hospital discharge. The colors of the quintiles correspond to colors as depicted in Fig. $2 \mathrm{~b}$. The highest UCE quintile had the best 5 -year survival, which declined with declining baseline UCE (log-rank test: $P<0.001$ ) 


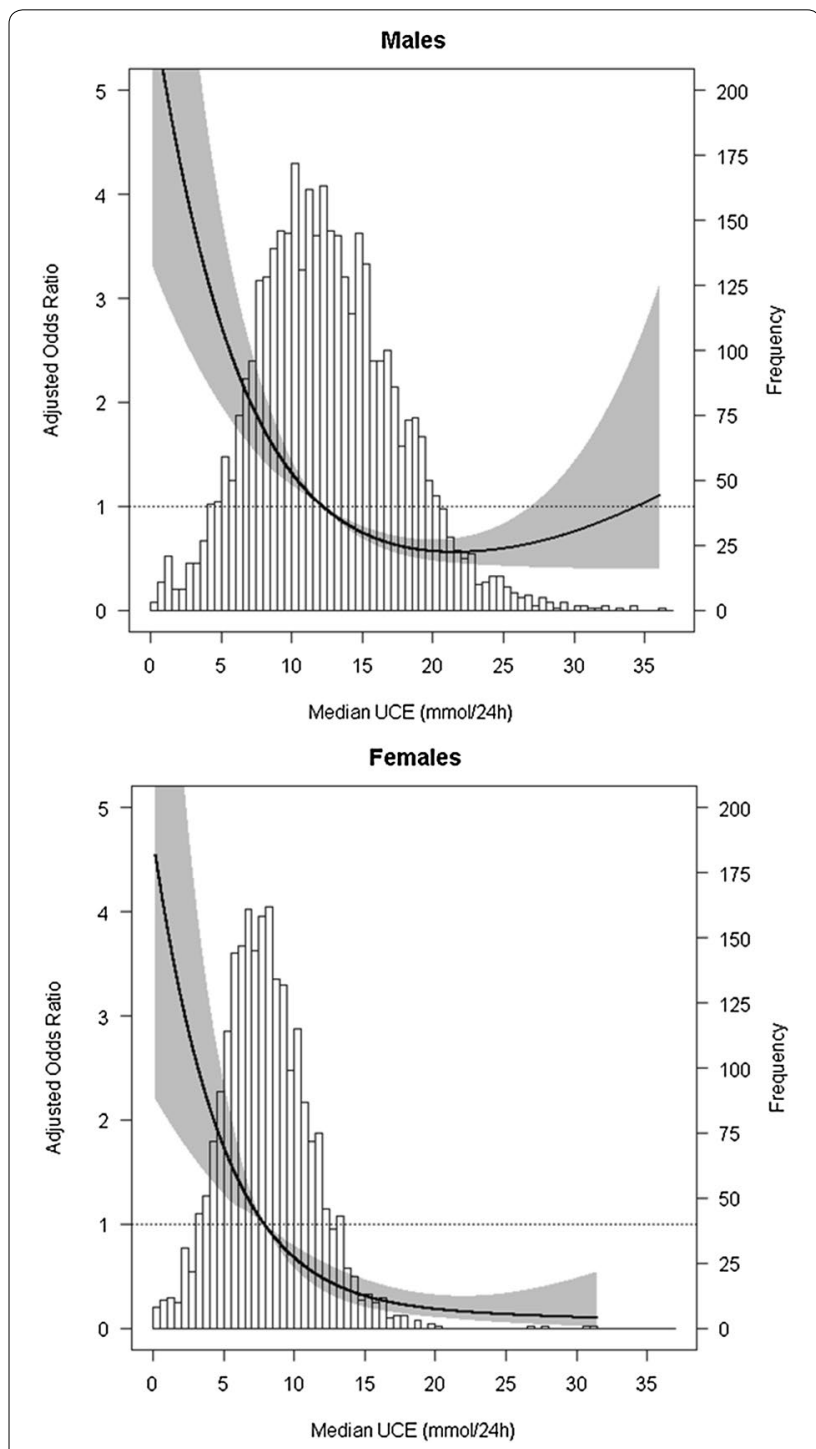

Fig. 3 Association between UCE and in-hospital mortality for both men and women. Data were fit by a multivariable logistic regression model based on restricted cubic splines. UCE was entered as continuous variable. Data were adjusted for sex, age, eGFR, BMI, severity of illness and reason of admission (model 5). Here, the median UCE was defined as the reference standard. The gray area represents the 95\% $\mathrm{Cl}$. Here, the median UCE was defined as the reference standard. The curves in particular underscore the inverse relation of UCE with mortality for low and near median UCE values. Note the widely diverging $95 \% \mathrm{Cl}$ at the extremes resulting from the low patient numbers and the cubic fit

Because of the known sex difference in UCE, multivariable adjusted restricted cubic splines for the association of UCE with in-hospital mortality are shown separately for men and women in Fig. 3.

\section{UCE and long-term mortality}

For the 5111 patients who were discharged alive from the hospital, long-term mortality was assessed. Overall 5-year mortality was 29\%. In univariate analysis, UCE showed a strong relation with long-term survival as illustrated by the Kaplan-Meier curves (log-rank test $P<0.001$, Fig. 2b). In Cox-regression with UCE expressed in quintiles, patients in the lowest UCE quintile had a four times higher risk for long-term mortality compared to those in the highest UCE quintile (HR 4.03, 95\% CI $3.35-4.84, P<0.001$, Table 3). After adjustment for potential confounders, this association remained independent (HR 2.32, 95\% CI 1.89-2.85, $P<0.001$, Table 3, model 5).

In Cox regression analysis with UCE expressed as a continuous variable, UCE was also associated with longterm mortality (HR 1.76, 95\% CI 1.66-1.88 for each $5 \mathrm{mmol} / \mathrm{d}$ decrease of UCE, $P<0.001$, Table 3$)$. This association remained independent after adjustment for confounders with an HR of 1.49 (95\% CI 1.38-1.62, $P<0.001$, Table 3, model 5).

A multivariable adjusted restricted cubic spline for the association between UCE and mortality over 5 years for both men and women is shown in Fig. 4.

\section{Subgroup, sensitivity and additional analyses}

Additional subgroup and sensitivity analyses concerning the role of AKI, BMI and rhabdomyolysis amongst others, are presented and shown in the SMF (Tables ST1ST10, Figures SF1-SF12). We found similar associations between UCE and short-term and long-term mortality in both the subgroup and sensitivity analyses. The association between UCE and short-term mortality was only not observed in trauma patients (OR 1.10, 95\% CI 0.71-1.71, $P=0.69$ ).

\section{Discussion}

This large prospective study shows that urinary creatinine excretion (UCE) early after ICU admission as a measure of muscle mass is strongly associated with both short-term and long-term mortality, independent of important covariates and confounders, including disease severity, age and renal function.

We consistently observed an inverse association between UCE and both short-term and long-term mortality, even in patients with chronic kidney disease or AKI (Tables ST1-ST10, Fig. SF4). Only for short-term outcome in trauma patients, no independent association with UCE was observed. However, a stronger association of UCE with long-term mortality was seen in the trauma patients when compared to the total patient group (Table ST4). As hospital mortality of severe trauma patients is mainly determined by age, severity of coma after trauma (and thus brain injury), base excess and 
Table 3 Cox proportional hazard regression analyses for $\mathbf{5}$-year mortality

\begin{tabular}{|c|c|c|c|c|c|c|c|}
\hline & UCE ${ }^{a}$ & & UCE sex-stratifi & yuintiles & & & \\
\hline & $(n=5111)$ & & Q1 $(n=850)$ & $Q 2(n=1006)$ & Q3 $(n=1040)$ & $Q 4(n=1092)$ & Q5 $(n=1123)$ \\
\hline & HR ( $95 \%$ Cl) & $P$ & $\mathrm{HR}(95 \% \mathrm{Cl})$ & HR $(95 \% \mathrm{Cl})$ & HR $(95 \%$ Cl) & HR $(95 \% \mathrm{Cl})$ & Reference \\
\hline Model $1^{\mathrm{b}}$ & $1.76(1.66-1.88)$ & $<0.001$ & $4.03(3.35-4.84)$ & $3.02(2.51-3.64)$ & $2.36(1.95-2.86)$ & $1.65(1.35-2.01)$ & 1.00 \\
\hline Model $2^{c}$ & $1.56(1.45-1.68)$ & $<0.001$ & $2.58(2.13-3.13)$ & $1.88(1.55-2.28)$ & $1.53(1.26-1.87)$ & $1.25(1.02-1.53)$ & 1.00 \\
\hline Model $3^{d}$ & $1.56(1.45-1.67)$ & $<0.001$ & $2.59(2.14-3.14)$ & $1.87(1.54-2.27)$ & $1.52(1.25-1.85)$ & $1.24(1.01-1.52)$ & 1.00 \\
\hline Model $4^{\mathrm{e}}$ & $1.56(1.45-1.68)$ & $<0.001$ & $2.59(2.12-3.17)$ & $1.87(1.53-2.29)$ & $1.52(1.24-1.85)$ & $1.24(1.01-1.51)$ & 1.00 \\
\hline Model $5^{f}$ & $1.49(1.38-1.62)$ & $<0.001$ & $2.32(1.89-2.85)$ & $1.71(1.39-2.09)$ & $1.39(1.13-1.70)$ & $1.17(0.95-1.43)$ & 1.00 \\
\hline
\end{tabular}

Cox proportional hazard regression analysis to assess the association of UCE with 5-year survival

a UCE was entered as a continuous variable per $5 \mathrm{mmol} / 24 \mathrm{~h}$ decrease

b Model 1: adjusted for sex in continuous analyses, no adjustment for sex-adjusted quintiles

c Model 2: adjusted as for model 1, additionally adjusted for age

d Model 3: adjusted as for model 2, additionally adjusted for kidney function (eGFR CKD-EPI)

e Model 4: adjusted as for model 3, additionally adjusted for body mass index (BMI)

${ }^{f}$ Model 5: adjusted as for model 4, additionally adjusted for severity of illness (APACHE-IV) and reason of admission (trauma vs non-trauma)

coagulation disturbances [15], UCE is likely to be only a minor determinant of the short-term prognosis of trauma patients.

The relation of UCE with mortality has already been established in several other patient groups. A higher mortality in patients with low (baseline) UCE is present in renal transplant patients [16], patients with stroke [17], coronary artery disease [8], heart failure [18] and chronic kidney disease [19]. Moreover, a similar association is observed in the general population [10]. We are the first to examine the relationship of UCE with mortality in a large heterogenic critically ill patient group.

In several ICU subgroups, a J-shaped association between BMI and mortality was shown [20]. Other studies also show a beneficial effect of a moderately elevated BMI in several patient groups, including the critically ill [21-26]. It is very plausible that the increased mortality of patients with a low BMI results from the adverse effects of sarcopenia, as we found the highest mortality risk in patients in the lowest UCE quintile after adjustment for BMI (Table ST6).

In patients without $\mathrm{AKI}$, a decreased serum creatinine also is a reflection of muscle wasting [27] and two large studies showed that low baseline serum creatinine is an independent risk factor for mortality [27, 28]. Changes in serum creatinine, i.e. in AKI patients, seem only to be associated with short-term mortality [29]. Prognostic ICU-models often incorporate serum creatinine as a measure of renal function [11,30]. Although the APACHE-IV score also considers a lowered serum creatinine level $\left(<53 \mu \mathrm{mol} \mathrm{L} \mathrm{L}^{-1}\right.$ or $\left.<0.6 \mathrm{mg} \mathrm{dL}^{-1}\right)$ a mortality risk [11], no prognostic ICU-scoring system utilizes $\mathrm{UCE}$ as an outcome predictor. Both serum creatinine and
UCE are influenced by renal insufficiency, but in steadystate conditions, urinary excretion will equal creatinine production, irrespective of the serum creatinine concentration. UCE will, therefore, better reflect muscle mass than serum creatinine, especially in patients with renal insufficiency. UCE determined early after ICU-admission might, therefore, improve prognostic ICU models and could be a significant contribution to the evolution of prognostic scores.

In our study we focused on UCE within 3 days of ICU admission and we did not focus on subsequent changes during ICU admission. In a recent study a decrease in UCE was seen after 7 and 14 days of ICU treatment, reflecting the gradual loss of muscle during ICU stay [31]. It seems plausible that a progressive decrease in UCE would further predict poor outcome, but this has to be assessed in future studies.

Although UCE presents a non-invasive and inexpensive method in ICU-patients, other methods of muscle mass estimation have been well researched in several patient populations; most are poorly suited for ICU patients [3237]. In the critically ill patient, anthropometric measurements such as body weight, BMI, waist circumference or mid-arm or mid-thigh muscle area are often complicated by the presence of dehydration, ascites or edema. More advanced techniques such as computed tomography, magnetic resonance imaging or dual-energy X-ray absorptiometry are both expensive and impractical for routine use in the ICU [5]. Bioelectrical impedance analysis is a simple and non-invasive method that is widely used to obtain estimates of body composition [38], but its accuracy in detecting loss of muscle mass in ICU patients is questionable because its measurement requires fluid 


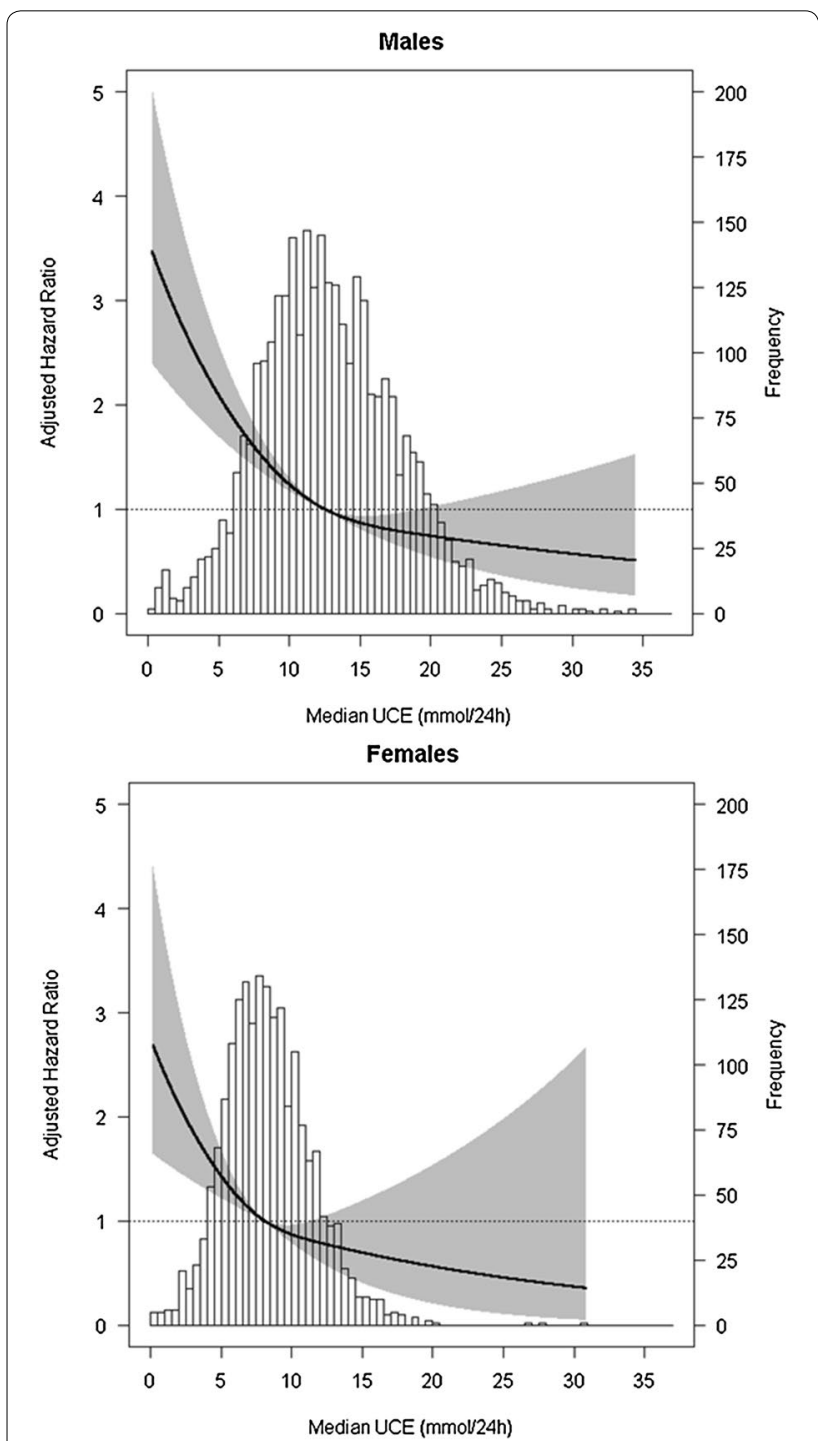

Fig. 4 Association between UCE and 5-year survival for both men and women discharged alive. Data were fit by a Cox proportional hazard regression model based on restricted cubic splines. UCE was entered as continuous variable. Data were adjusted for sex, age, eGFR, $B M I$, severity of illness and reason of admission (model 5). The gray area represents the $95 \% \mathrm{Cl}$

homeostasis [39]. Repeated ultrasonography for the detection of muscle wasting shows promising results in a few relatively small studies [33-35], but muscle dimensions are also influenced by generalized edema. In this regard, it would be interesting to compare UCE with both bioelectrical impedance and ultrasonography in a larger study population, while taking the fluid balance into account.

Some limitations of our study are due to its post hoc design and the long period it covers. An important potential limitation of UCE are the rapid changes in glomerular filtration rate as are common in the critically ill [40, 41]. UCE may decrease in patients with acute kidney injury, who have a higher risk of dying [30]. In some cases, UCE may increase because of augmented renal clearance, as has been reported in some younger trauma and sepsis patients [40]. Also, glomerular filtration may be altered by commonly administered drugs, such as vasopressors and diuretics $[42,43]$. The differences in mortality could thus possibly be attributed to other factors such as renal function or hypercatabolism instead of muscle mass. We were unfortunately not able to address this as we did not perform true GFR measurements or other muscle mass measurements.

The relevance of decreased or increased glomerular filtration or creatinine clearance with respect to UCE could best be addressed by determining this parameter as well. We did adjust for eGFR as potential confounder; however, in the case of AKI it may take time before serum creatinine rises, limiting the value of this adjustment. However, mean UCE did not significantly differ between days 1 and 3, and we excluded patients with severe AKI (stage 3). Furthermore, separate analyses performed for both patients without and with AKI stage 1 or 2 (SMF: Table ST3a, ST3b, Fig. SF4) led to similar findings. Finally, we excluded patients with AKI stage 3, also because UCE cannot be determined in anuric patients. This is an obvious limitation of using this marker as a prognostic score. Estimation of muscle mass by measurement of UCE also requires complete $24 \mathrm{~h}$ urine collection by ICU-nurses. Since ICU patients typically have indwelling urine catheters, this was an advantage in our population. In non-ICU patients who often have to collect the 24-h urine themselves, it is therefore considered a less reliable method [4]. Creatinine levels in patients who are on an oral diet may also be increased by meat intake. In our study, this potential confounding factor was of no influence since all patients were on enteral or parenteral feeding containing no dietary meat. Our population consists of predominantly surgical rather than medical ICU patients. However, we saw similar findings in the population of non-surgical ICU patients (See SMF) and UCE was found to be a strong predictor of mortality in non-ICU medical patients as well [10, 17-19]. Recently, the sarcopenia index has been proposed as a measure for muscle mass [36, 37]. Although promising, we were unable to use this index as it requires cystatine $C$ measurement. Due to the retrospective nature of our study we were not able to compare UCE with other methods that estimate muscle mass, i.e. the paraspinal muscle surface area at lumbal vertebral levels measured on CT [5,37]. However, future studies could assess the relationship between UCE and other muscle mass measures. 
In conclusion, low urinary creatinine excretion early after ICU admission is a strong independent predictor of both short-term and long-term mortality after adjustment for BMI, renal function and severity of disease, underscoring a role of muscle mass as risk factor for mortality. UCE thus constitutes a simple, readily available and relevant prognostic biomarker for critically ill patients.

\section{Electronic supplementary material}

The online version of this article (https://doi.org/10.1007/s00134-018-5359-6) contains supplementary material, which is available to authorized users.

\section{Author details}

${ }^{1}$ Department of Critical Care, University of Groningen, University Medical Center Groningen, Hanzeplein 1, 9700 RB Groningen, The Netherlands. 2 Department of Intensive Care, Medical Center Leeuwarden, Leeuwarden, The Netherlands. ${ }^{3}$ Department of Nephrology, University of Groningen, University Medical Center Groningen, Groningen, The Netherlands. ${ }^{4}$ Department of Intensive Care Medicine, VU University Medical Center, Amsterdam, The Netherlands.

\section{Acknowledgements}

We thank Wim Dieperink, PhD, of the Department of Critical Care, University Medical Center Groningen, for administrative support. We also thank Leendert H. Oterdoom, MD PhD, of the Department of Gastroenterology and Hepatology, VU University Medical Center, for reviewing and commenting on earlier drafts of the manuscript.

\section{Funding}

There was no external funding for this work.

\section{Compliance with ethical standards}

\section{Conflicts of interest}

The authors declare that they have no competing interests.

\section{Open Access}

This article is distributed under the terms of the Creative Commons Attribution-NonCommercial 4.0 International License (http://creativecommons.org/ licenses/by-nc/4.0/), which permits any noncommercial use, distribution, and reproduction in any medium, provided you give appropriate credit to the original author(s) and the source, provide a link to the Creative Commons license, and indicate if changes were made.

Received: 10 May 2018 Accepted: 28 August 2018

Published online: 7 September 2018

\section{References}

1. Puthucheary ZA, Rawal J, McPhail M et al (2013) Acute skeletal muscle wasting in critical illness. JAMA 310:1591-1600

2. Moisey LL, Mourtzakis M, Cotton BA et al (2013) Skeletal muscle predicts ventilator-free days, ICU-free days, and mortality in ICU patients. Crit Care 17:R206

3. Weijs PJM, Looijaard WGPM, Dekker IM, Stapel SN, Girbes AR, Oudemans van Straaten HM, Beishuizen A (2014) Low skeletal muscle area is a risk factor for mortality in mechanically ventilated critically ill patients. Crit Care 18:R12

4. Beddhu S, Pappas LM, Ramkumar N, Samore M (2003) Effects of body size and body composition on survival in hemodialysis patients. J Am Soc Nephrol 14:2366-2372

5. Proctor DN, O'Brien PC, Atkinson EJ, Nair KS (1999) Comparison of techniques to estimate total body skeletal muscle mass in people of different age groups. Am J Physiol Endocrinol Metab 277:E489-E495
6. Wyss M, Kaddurah-Daouk R (2000) Creatine and creatinine metabolism. Physiol Rev 80:1107-1213

7. Heymsfield SB, Arteaga C, McManus C, Smith J, Moffitt S (1983) Measurement of muscle mass in humans: validity of the 24-h urinary creatinine method. Am J Clin Nutr 37:478-494

8. Ix JH, de Boer IH, Wassel CL, Criqui MH, Shlipak MG, Whooley MA (2010) Urinary creatinine excretion rate and mortality in persons with coronary artery disease: the heart and soul study. Circulation 121:1295-1303

9. Baxmann AC, Ahmed MS, Marques NC et al (2008) Influence of muscle mass and physical activity on serum and urinary creatinine and serum cystatin C. Clin J Am Soc Nephrol 3:348-354

10. Oterdoom LH, Gansevoort RT, Schouten JP, de Jong PE, Gans ROB, Bakker SJL (2009) Urinary creatinine excretion, an indirect measure of muscle mass, is an independent predictor of cardiovascular disease and mortality in the general population. Atherosclerosis 207:534-540

11. Zimmerman JE, Kramer AA, McNair DS, Malila FM (2006) Acute physiology and chronic health evaluation (APACHE) IV: hospital mortality assessment for today's critically ill patients. Crit Care Med 34:1297-1310

12. Ja Kellum, Lameire N (2013) Diagnosis, evaluation, and management of acute kidney injury: a KDIGO summary (Part 1). Crit Care 17:2014

13. Janssen I, Heymsfield SB, Wang Z, Ross R (2000) Skeletal muscle mass and distribution in 468 men and women aged 18-88 year. J Appl Physiol 89:81-88

14. Levey AS, Stevens AL, Schmid CH et al (2009) A new equation to estimate glomerular filtration rate. Ann Intern Med 150:604-612

15. Raum MR, Nijsten MW, Vogelzang M et al (2009) Emergency trauma score: an instrument for early estimation of trauma severity. Crit Care Med 37:1972-1977

16. Oterdoom LH, van Ree RM, de Vries APJ et al (2008) Urinary creatinine excretion reflecting muscle mass is a predictor of mortality and graft loss in renal transplant recipients. Transplantation 86:391-398

17. Hsu CY, Wu YL, Cheng CY et al (2015) Low baseline urine creatinine excretion rate predicts poor outcomes among critically ill acute stroke patients. Curr Neurovasc Res 12:47-52

18. Ter Maaten JM, Damman K, Hillege HL, Bakker SJ, Anker SD, Navis G, Voors AA (2014) Creatinine excretion rate, a marker of muscle mass, is related to clinical outcome in patients with chronic systolic heart failure. Clin Res Cardiol 103:976-983

19. Wilson FP, Xie D, Anderson Ah et al (2014) Urinary creatinine excretion, bioelectrical impedance analysis, and clinical outcomes in patients with CKD: the CRIC study. Clin J Am Soc Nephrol 9:2095-2103

20. Pickkers P, de Keizer N, Dusseljee J, Weerheijm D, van der Hoeven JG, Peek $N$ (2013) Body mass index is associated with hospital mortality in critically ill patients: an observational cohort study. Crit Care Med 41:1878-1883

21. Hutagalung R, Marques J, Kobylka K, Zeidan M, Kabisch B, Brunkhorst F, Reinhart K, Sakr Y (2011) The obesity paradox in surgical intensive care unit patients. Intensive Care Med 37:1793-1799

22. Flegal KM, Kit BK, Oprana H, Graubard BI (2013) Association of all-cause mortality with overweight and obesity using standard body mass index categories: a systematic review and meta-analysis. JAMA 309:71-82

23. Abhyankar S, Leishear K, Callaghan FM, Demner-Fushman D, McDonald CJ (2012) Lower short- and long-term mortality associated with overweight and obesity in a large cohort study of adult intensive care unit patients. Crit Care 16:R235

24. Sakr Y, Alhussami I, Nanchal R, Wunderink R, Pellis T, Wittebole X, MartinLoeches I, Francois B, Leone M, Vincent JL (2015) Being overweight is associated with greater survival in ICU patients: results from the intensive care over nations audit. Crit Care Med 42:2623-2632

25. Pepper DJ, Sun JF, Welsh J, Cui XZ, Suffredini AF, Eichacker PQ (2016) Increased body mass index and adjusted mortality in ICU patients with sepsis or septic shock: a systematic review and meta-analysis. Crit Care 20:181

26. Hartrumpf M, Kuehnel RU, Albes JM (2017) The obesity paradox is still there: a risk analysis of over 15000 cardiosurgical patients based on body mass index. Interact Cardiovasc Thorac Surg 25:18-24

27. Cartin-Ceba R, Afessa B, Gajic O (2007) Low baseline serum creatinine concentration predicts mortality in critically ill patients independent of body mass index. Crit Care Med 35:2420-2423

28. Udy AA, Scheinkestel C, Pilcher D et al (2016) The association between low admission peak plasma creatinine concentration and in-hospital mortality in patients admitted to intensive care in Australia and New Zealand. Crit Care Med 44:73-82 
29. Mildh H, Pettilä V, Korhonen A-M, Karlsson S, Ala-Kokko T, Reinikainen M Vaara ST (2016) Three-year mortality in 30-day survivors of critical care with acute kidney injury: data from the prospective observational FINNAKI study. Ann Intensive Care 6:118

30. Ferreira FL, Bota DP, Bross A, Melot C, Vincent JL (2001) Serial evaluation of the SOFA score to predict outcome in critically ill patients. JAMA 286:1754-1758

31. Schetz M, Gunst J, van den Berghe G (2014) The impact of using estimated GFR versus creatinine clearance on the evaluation of recovery from acute kidney injury in the ICU. Intensive Care Med 40:1709-1717

32. Pahor M, Manini T, Cesari M (2009) Sarcopenia: clinical evaluation, biological markers and other evaluation tools. J Nutr Health Aging 13:724-728

33. Cartwright MS, Kwayisi G, Griffin LP (2013) Quantitative neuromuscular ultrasound in the intensive care unit. Muscle Nerve 47:255-259

34. Connollu B, MacBean V, Crowley C, Lunt A, Moxham J, Rafferty GF, Hart N (2015) Ultrasound for the assessment of the peripheral skeletal muscle architecture in critical illness: a systematic review. Crit Care Med 43:897-905

35. Campbell IT, Watt T, Withers D, England R, Sukumar S, Keegan MA (1995) Muscle thickness, measured with ultrasound, may be an indicator of lean tissue wasting in multiple organ failure in the presence of edema. Am J Clin Nutr 62:533-539

36. Kashani KB, Frazee EN, Kukrálová L, Sarvottam K, Herasevich V, Young PM, Kashyap R, Lieske JC (2017) Evaluating muscle mass by using markers of kidney function: development of the sarcopenia index. Crit Care Med 45:e23-e29
37. Kashani KB, Sarvottam K, Pereira NL, Barreto EF, Kennedy CC (2018) The sarcopenia index: a novel measure of muscle mass in lung transplant candidates. Clin Transpl 32(3):e12182

38. Kuchnia A, Earthman C, Teigen L et al (2016) Evaluation of bioelectrical impedance analysis in critically ill patients: results of a multicenter prospective study. J Parenter Enter Nutr 41(7):1131-1138

39. Forni LG, Hasslacher J, Joannidis M (2015) Bioelectrical impedance vector analysis in the critically ill: cool tool or just another toy? Crit Care 19:387

40. Udy AA, Roberts JA, Shorr AF et al (2013) Augmented renal clearance in septic and traumatized patients with normal plasma creatinine concentrations: identifying at-risk patients. Crit Care 16:R35

41. Udy AA, Baptista JP, Lim NL et al (2014) Augmented renal clearance in the ICU: results of a multicenter observational study of renal function in critically ill patients with normal plasma creatinine concentration. Crit Care Med 42:520-527

42. Bellomo R, Kellum JA, Ronco C, Wald R, Martensson J, Maiden M, Bagshaw SM, Glassford NJ, Lankadeva Y, Vaara ST, Schneider A (2017) Acute kidney injury in sepsis. Intensive Care Med 41:816-828

43. Lankadeva YR, Kosaka J, Evans RG, Bailey SR, Bellomo R, May CN (2016) Intrarenal and urinary oxygenation during norepinephrine resuscitation in ovine septic acute kidney injury. Kidney Int 90:100-108 